\title{
Example-based Reflectance Estimation for Capturing Relightable Models of People
}

\author{
P. Stroia-Williams ${ }^{1}$, A. Hilton 1 \\ ${ }^{1}$ University of Surrey, CVSSP, University of Surrey, Guildford
}

Keywords: Relighting, reflectance estimation, non-parametric statistics.

\begin{abstract}
We present a new approach to reflectance estimation for dynamic scenes. Non-parametric image statistics are used to transfer reflectance properties from a static example set to a dynamic image sequence. The approach allows reflectance estimation for surface materials with inhomogeneous appearance, such as those which commonly occur with patterned or textured clothing. Material reflectance properties are initially estimated from static images of the subject under multiple directional illuminations using photometric stereo. The estimated reflectance together with the corresponding image under uniform ambient illumination form a prior set of reference material observations. Material reflectance properties are then estimated for video sequences of a moving person captured under uniform ambient illumination by matching the observed local image statistics to the reference observations. Results demonstrate that the transfer of reflectance properties enables estimation of the dynamic surface normals and subsequent relighting. This approach overcomes limitations of previous work on material transfer and relighting of dynamic scenes which was limited to surfaces with regions of homogeneous reflectance. We evaluate for relighting 3D model sequences reconstructed from multiple view video. Comparison to previous model relighting demonstrates improved reproduction of detailed texture and shape dynamics.
\end{abstract}

\section{Introduction}

This paper presents a new approach to reflectance estimation for video sequences of dynamic deformable surfaces. Our approach allows relightable model capture techniques previously limited to uniform colour surfaces[11, 15] to be applied to textured surfaces.

Previous approaches for capturing reflectance of dynamic deformable subjects have made use of illumination maps and surface shape data allowing the surface reflectance to be calculated directly from the diffuse reflectance model $[3,10]$. Such an approach depends on the accuracy of the illumination map and surface shape data. In particular estimation of accurate surface normals is critical to recovery of surface reflectance properties. The resultant reflectance map may be contaminated by any shading not accounted for by the illumination model and surface shape data. In Section 5.1 we compare our approach with de-lighting as part of a relightable model capture framework.

Other methods use observations of each surface point under multiple illuminations allowing photometric stereo (PS) to be applied. This requires either the subject to remain static whilst the illumination changes or for the surface points to be tracked. Tracking to the required dense coverage is an unsolved problem for deformable surface without known texture. Results have been shown for this approach by using a generic human model [18], but this does not track the true shape of the subject leading to biasing of the large-scale shape. Recent work [12] performed photometric stereo for dynamic scenes using simultaneous capture under multiple coloured illuminations. However, this approach requires the assumption of a Lambertian surface with uniform albedo.

Detailed dynamic shape has been successfully captured using standard studio lighting and cameras using shapefrom-shading based approaches to estimate the surface normal[15, 11]. However these approaches are limited to cloth materials with uniform colour regions. The reflectance estimation approach introduced in this paper allows estimating of surface shading for materials with non-uniform reflectance properties. Subsequent estimation of surface normals and transfer of material reflectance properties allows relighting of dynamic scenes with textured and patterned surface appearance.

Our reflectance capture method makes use of the image based non-parametric statistics. Non-parametric statistics have been recently used to represent the local image appearance structure in a number of contexts: image analogies [13]; image infilling [4]; and view-interpolation [19]. Non-parametric statistics provide a general representation of the local structure observed in an image region without a prior model or assumptions on appearance, allowing indexing to identify similar regions. In this work non-parametric statistics are used to represent the reflectance properties of a static scene captured under multiple illumination conditions. The reflectance properties are then transferred to novel image observations in video sequences by searching for reference image regions with similar local appearance statistics. This allows us to potentially bring the accuracy of the static only methods to dynamic sequences.

In Section 2 we review related research on multiple view reconstruction and relighting of dynamic scenes. Section 3 presents an overview of the approach to reflectance transfer 


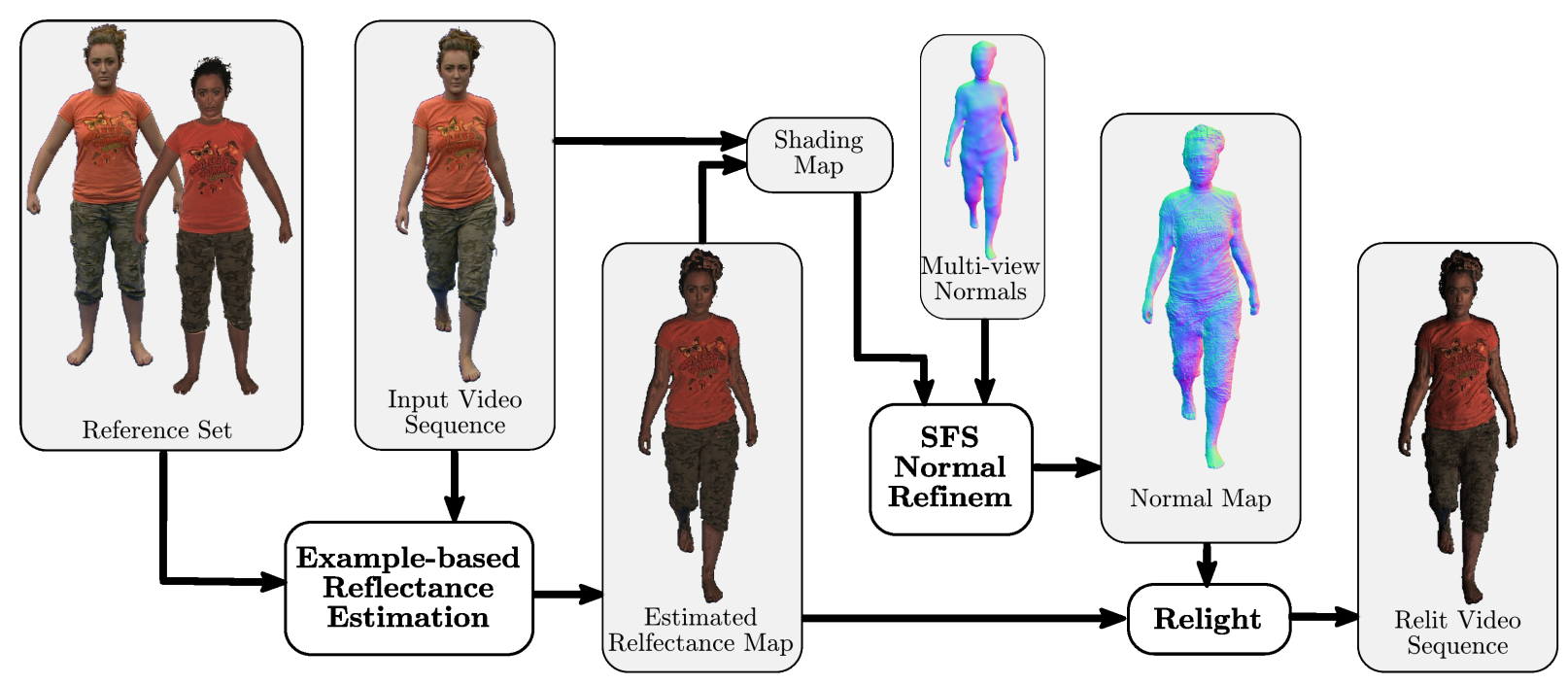

Figure 1: Relighting Process Overview

for dynamic scenes introduced in this paper. The methodology for reflectance estimation and transfer is then presented in Section 4. Results and a comparative evaluation with existing approaches for relighting dynamic scenes of people is presented in Section 5 followed by conclusions in Section 6 .

\section{Related Work}

Over the past decade there has been extensive interest in performance reconstruction from multiple view video for freeviewpoint $3 \mathrm{D}$ video replay. Research has primarily focused on accurate reconstruction of shape and high-quality rendering of novel views of the captured performance. Reuse for entertainment production in film, broadcast or games often requires relighting to composite the captured performance within a novel scene. Photo-realistic relighting of dynamic scenes remains a challenging open-problem. In this section we review research related to the problem in the estimation of surface reflectance properties and appearance transfer to manipulate dynamic scene appearance.

\subsection{Reflectance Estimation and Relighting Dynamic Scenes}

Estimation of surface reflectance properties from image observations is a classical problem in computer vision. In general the observed surface appearance depends on the illumination, surface reflectance and surface shape and orientation (normal). Reconstruction of the surface reflectance is an ill-conditioned inverse problem which requires accurate knowledge of both surface orientation and illumination. Photo-metric stereo [2] allows the estimation of the surface albedo or diffuse component for Lambertian surfaces from three or more images of the object taken under different point light sources with known position and intensity. Acquisition of the surface with multiple light sources requires a static scene to recover the albedo independently for each pixel.
Photo-metric stereo has recently been used to recover per pixel surface normals using simultaneous acquisition with multiple coloured lights from different directions [12]. This approach is limited to Lambertian surfaces with uniform albedo restricting its application to real scenes. Estimation of both diffuse and specular surface reflectance from multiple view capture has been achieved using approximate temporal correspondence to obtain multiple observations of surface regions with uniform albedo over time [18]. However, surface tracking is not sufficiently effective for this approach without using simplified models that lead to significant biasing in the large-scale shape.

Recovery of parametric models of surface reflectance properties for static objects has been achieved using an accurate prior measurement of surface shape obtained from range scans [16]. For dynamics scenes accurate reconstruction of fine surface detail from multiple view images or videorate range scans remains an open problem. Fine detail such as creases in clothing is typically not reproduced in surface reconstructions of a person [17] resulting in surface normals with insufficient accuracy to recover the reflectance. Relighting of large scale structures assuming Lambertian reflectance can be achieved by estimating the albedo given inaccurate estimates of shape and orientation [10]. These approaches enable large scale relighting of dynamic scenes but do not relight fine detail such as wrinkles in clothing. An alternative approach to relighting dynamic scenes recently proposed uses detailed normal estimates from surface shading [15]. This approach assumes surface regions with uniform Lambertian reflectance which are segmented to obtain a single albedo estimate for each region. Given the albedo estimate the shading within each region is used to estimate the local surface orientation and illumination direction, Estimation is regularised using a prior coarse model of surface shape reconstructed from multiple views. The approach achieves realistic relighting of clothing detail such as creases but is limited to clothing with uniform appearance. 
Image-based relighting uses images of the scene captured under a large number of illumination conditions to produce images with complex illuminations by combining the captured images. This approach has been applied to dynamic scenes using a light-stage with high-speed $(4000 \mathrm{~Hz})$ switching of illumination sources and image capture [6]. Results demonstrate photo-realistic video sequences of performers under novel illumination conditions. The use of specialist lighting and high-frame rate cameras limits the wide-spread application of this approach.

\subsection{Appearance Transfer}

Manipulation of dynamic scene appearance in images and video has been investigated for editing texture appearance $[7,8]$ and material properties [14]. The approaches use normal from shading to estimate the local surface orientation to modify the image appearance. Fang and Hart [8] transfer novel texture map appearance into video sequences using temporal tracking and shape-from-shading to estimate orientation. However, these approaches do not address the problem of relighting in images.

In this paper we propose a technique to transfer the material properties from reference images with known albedo to a video sequence. Lambertian reflectance properties for reference images are estimated using photometric stereo on a set of images captured in a static pose. Mapping of reflectance properties from the reference image to the novel image sequence of a dynamic scene is based on matching local image structure. Block based representation and matching of image structure has previously been used for image synthesis, infilling and view-interpolation [13, 5, 9].

Local image blocks provide a non-parametric representation of the image statistics which can be used as a prior to constrain the synthesis of novel image patches. In this paper we demonstrate that an analogous approach can be used to transfer material properties from a reference image to a video sequence captured under the same illumination conditions.

\section{Overview}

An overview of the reflectance transfer method for relighting is presented in Figure 1. As inputs we take a set of reference data $\mathbb{I}$ and a video stream of the actors performance $D_{l}$. The reference set $\mathbb{I}$ comprises of diffuse albedo maps $A_{s}$ and their associated image $R_{s}$ captured under the same conditions as the performance $D_{l}$. In this work the reference material reflectance properties are reconstructed using photometric stereo on images of the subject captured in a static pose under multiple directional illuminations. Care is taken to ensure the actor holds the same pose whilst $\mathbb{I}$ is captured in order to ensure pixel-wise correspondence between the images.

A dynamic albedo map $D_{a}$ is estimated from the video sequences $D_{l}$ of the subjects performance by transfer of the reference material properties using the reference images $R_{s}$ of the static subject acquired under the same illumination conditions. For each pixel in each frame of $D_{l}$ we search each $R_{s}$ for the best match of local image structure. The corresponding albedo from $A_{s}$ is then transferred to estimate an albedo for each pixel giving a video albedo map image sequence $D_{a}$. The estimated albedo map forms the basis for estimating the dynamic surface normal at each pixel in the input image sequences and relighting the sequence to produce a novel relit video sequence $D_{r}$.

\section{Reflectance Estimation for Dynamic Scenes}

Given a video image sequence $D_{l}$ of a dynamic scene captured under unknown illumination conditions the problem is to relight the scene to produce a sequence $D_{r}$ under novel illumination conditions. Assuming Lambertian surface reflectance the observed reflected radiance for a surface point $D_{l}(i, j, t)=\left(r_{i j t}, g_{i j t}, b_{i j t}\right)$ for the surface point corresponding to pixel $(i, j)$ at time $t$ is given by:

$$
D_{l}(i, j, t)=a_{i j t} \int_{\vec{\phi}} \vec{L}_{i j t}(\vec{\phi})\left(\vec{n}_{i j t} \cdot \vec{\phi}\right) d \vec{\phi}
$$

where $a=\left(a_{i j t}^{r}, a_{i j t}^{g}, a_{i j t}^{b}\right)$ is the surface albedo, $\vec{n}_{i j t}$ is the surface normal and $\vec{L}(\vec{\phi})$ is the incident irradiance in direction $\phi$ which is integrated over the hemisphere in the positive normal direction. In general both the surface normal, albedo and irradiance are unknown. The irradiance changes across the surface according to both the scene illumination and surface visibility due to self-shadow.

In general estimation of the albedo at a surface point requires accurate knowledge of the surface normal, scene illumination and object shape to evaluate self-shadowing. For non-rigid dynamic scenes it is not possible to simultaneously capture the scene under multiple directional illuminations. Previous research on relighting dynamic scenes [15] has assumed uniform Lambertian surface regions and a single point light source, allowing segmentation to estimate the region albedo followed by estimation of the surface normal at each point. In this work the objective is to relight surfaces with unknown non-uniform albedo and unrestricted scene illumination. To achieve this objective surface reflectance properties are estimated from initial observations of the subject under multiple illumination conditions using photo-metric stereo. The estimated reflectance properties are then transferred to image sequence observations of a dynamic scenes by matching the observations in the static scene.

\subsection{Initial Estimation of Material Properties}

Photo-metric stereo [2] is used to estimate the surface normal and albedo of the scene assuming Lambertian reflectance. For each pose $s$ of the subject we capture a set of images $\left\{I_{i j}\right\}_{i=1}^{n_{i}}$ under known point light source illuminations $L_{i}$ together with an image $R_{s}$ under the same illumination conditions as the dynamic capture. Photometric stereo gives an estimate albedo image $A_{s}$ and normal image $N_{s}$. The initial estimate of 
material properties using photometric stereo gives a set $\mathbb{I}=$ $\left\{R_{s}, A_{s}, N_{s}\right\}_{s=1}^{N_{I}}$ of reference images $R_{s}$ and corresponding albedo $A_{s}$ and normal $N_{s}$ images for each pose $s$. This forms the basis for transferring material properties to the dynamic scene observed under the same illumination conditions as the reference image set $\left\{R_{s}\right\}$.

Our photo-metric stereo method follows Barsky's colour photo-metric stereo method [2]. The intensity values $\overrightarrow{l_{i}}=\left(l_{i}^{r}, l_{i}^{g}, l_{i}^{b}\right)$ observed under illuminations $L_{i}$ must be split into colour $\vec{C}_{i}$ and intensity values $S_{i}$ such that the error between the left hand side and right hand side of Equation 2 is minimised. We do this using a least squares approach.

$$
\mathbb{L}=\left[\begin{array}{ccc}
l_{1}^{r} & l_{1}^{g} & l_{1}^{b} \\
\vdots & \vdots & \vdots \\
l_{i}^{r} & l_{i}^{g} & l_{i}^{b}
\end{array}\right] \simeq\left[\begin{array}{ccc}
S_{1} C_{1}^{r} & S_{1} C_{1}^{g} & S_{1} C_{1}^{b} \\
\vdots & \vdots & \vdots \\
S_{i} C_{i}^{r} & S_{i} C_{i}^{g} & S_{i} C_{i}^{b}
\end{array}\right]
$$

Then, grayscale photo-metric stereo can be applied to the intensity part.

$$
a \vec{n}=\left([\mathbb{L}]^{T}[\mathbb{L}]\right)^{-} 1[L]^{T} \vec{S}
$$

Where $a=|\vec{a}|$ is the grayscale albedo and the colour albedo $\vec{a}=a \vec{c}$.

In order to capture this reflectance set we used 5 standard studio spot lights and a studio quality HD video camera along with the lighting setup for the dynamic capture. The spot lights were arranged with with 4 roughly in a circle around video camera and the 5th alongside the camera. The directions of the lights to the center of the capture volume relative to the camera were calibrated using an image of the setup captured with a Spheron HDR camera from the center of the capture volume. Any camera with known intrinsics would be suitable. From this image we manually selected the centers of the spot lights and camera allowing the directions from the center of the capture volume to be calculated. We calibrated the intensity of the spot lights using a diffuse sphere placed in the center of the capture volume. We compared the maximum intensity for each spot light as observed by the video camera and equalized their intensity. We have tried using the spot lights at their maximum settings and compensating in the photometric stereo calculation instead but found this to be less accurate and made use of less of the cameras dynamic range.

To capture the required images we asked the actor to stand still in the center of the capture volume as the 5 spot lights and dynamic capture studio lights cycled though, giving 5 illumination conditions for the photometric stereo calculation and the illumination under which the dynamic sequences will be captured. The total sequence takes less then 10 seconds to capture with the limiting factor being the fade in time for the spot lights.

\subsection{Example-based Albedo Transfer}

Estimation of the surface albedo for dynamic scenes with unknown surface orientation and illumination is based on the transfer of albedo estimates from the reference examples of
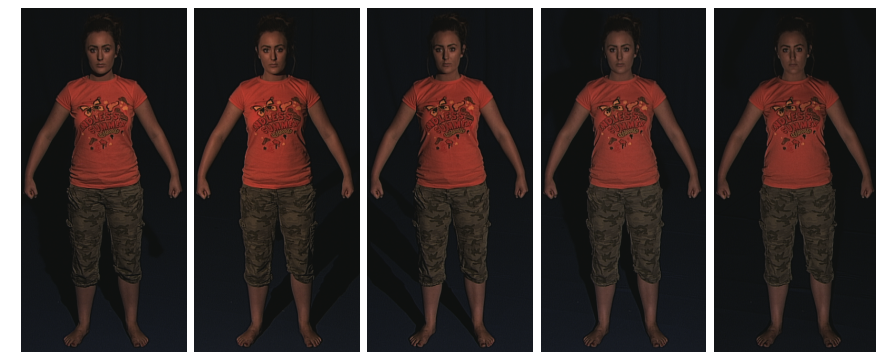

(a) Input Images for photo-metric stereo

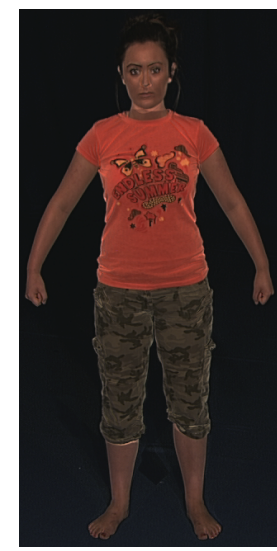

(b) Reference Albedo

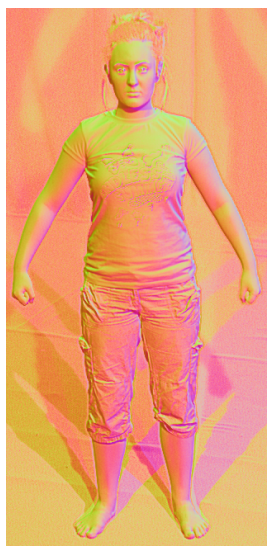

(c) Referecne Normal

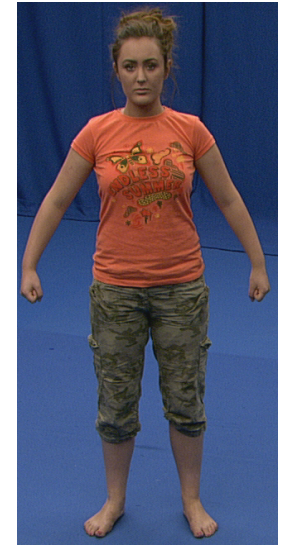

(d) Reference ambient lighting
Figure 2: Example reference set.

the same subjects which include images captured under the same illumination conditions as the dynamic scene. Transfer is based on the similarity of local image structure in the dynamic and reference images. This approach is similar to the nonparametric representation of image structure used in previous work for image analogies, infilling and view interpolation $[13,4,19]$.

In this work for each pixel in the observed image at time $t, D_{l}(i, j, t)$, we estimate the albedo by finding the region in the reference image set that, $R_{s}(p, q)$, that minimises the difference in local image appearance, $\mathrm{d}()$ :

$$
\begin{array}{r}
A(i, j, t)=A_{s}(p, q): \arg \min _{p, q, s} d\left(D(i, j, t), I_{s}(p, q)\right), \\
\left(I_{s}, A_{s}\right) \in \mathbb{I}, s \in\left[1, N_{I}\right],(p, q) \in[1, N]
\end{array}
$$

where $d\left(I_{1}(i, j), I_{2}(p, q)\right)$ is a measure of the local image difference for a region around the pixel $(i, j)$ in image $I_{1}$ and pixel $(p, q)$ in image $I_{2}$. The underlying assumption of this approach is that the reference image set contains examples of the reference material with similar local illumination, orientation and shape. A single reference image of clothing acquired under the same illumination as the dynamic scene commonly contains multiple examples of the same material with different orientations and local surface deformation 
providing a set of exemplars for matching, A Lambertian reflectance model is assumed as in previous approaches to relighting dynamic scenes. In the case of non-Lambertian materials the specular component will be treated as part the diffuse appearance requiring an increased number of exemplars for matching. This will also result in incorrect surface normal estimates and relighting. The algorithm for albedo transfer is presented in Algorithm 1, for each pixel in the observed dynamic scene $D_{l}(i, j, t)$ we search for the exemplar with minimum difference in appearance from the reference image set as defined by equation 4 . The corresponding albedo from the reference image $A_{s}(p, q)$ is then transferred to the dynamic scene pixel $D_{a}(i, j, t)$.

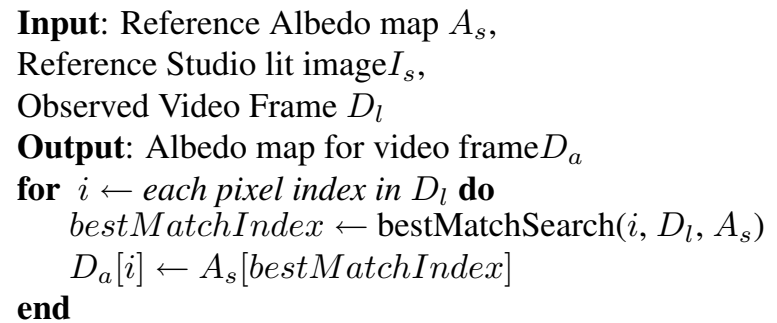

Algorithm 1: Example based reflectance transfer

\subsection{Non-parametric matching}

The function bestMatchSearch in Algorithm 1 which evaluates the exemplar with minimum difference $d()$ from all exemplars in the reference set $\mathbb{I}$ is critical to the quality of the results. As the actor moves around the scene the surface illumination will change due to variations in illumination in the scene, changes in surface orientation within the illumination field, self shadowing and inter-reflection. We seek to compensate for these affects as well as those due to noise through our choice of best match metric.

We use a normalised sum of squared distance (NSSD) metric which scales the pixel colour values by the average intensity within an $n \times n$ window around the pixel $(i, j)$. This metric removes global intensity difference between the observed and reference image windows to measure the difference in the local colour image structure. Normalising by the average intensity removes the effect of large scale illumination variations across the entire window. The NSSD metric for an $n \times n$ window is applied in RGB colour space as follows:

$$
\begin{aligned}
& d_{N S S D}\left(D(i, j, t), R_{s}(p, q)\right)= \\
& \sum_{u=-\frac{n}{2}}^{\frac{n}{2}} \sum_{v=-\frac{n}{2}}^{\frac{n}{2}}\left[\frac{D(i+u, j+v, t)}{\bar{D}}-\frac{R_{s}(p+u, q+v)}{\bar{R}_{s}}\right]^{2}
\end{aligned}
$$

where $D(i, j, t)$ and $R_{s}(p, q)$ are the rgb colour vectors in the observed and reference image respectively. $\bar{D}$ and $\bar{R}_{s}$ are the average intensity values over the $n \times n$ window.

Search for the exemplar patch which minimises the NSSD distance $d_{N} S S D()$, defined by equation 5 , for each pixel in the observed image has a computational complexity of $O\left(n^{2} N^{4} N_{I}\right)$ where $n$ is the window size, $N$ is number of pixels in the reference images and $N_{I}$ is the number of reference images. Full search is therefore prohibitively expensive. Instead we compromise optimality for speed using the off the shelf ANN (Approximate Nearest Neighbour) library[1]. For our case ANN finds the nearest neighbour with an error $\leq e$ with a computational complexity of $O\left(c_{e} \log \left(n N N_{I}\right)\right)$, where $c_{e}$ is a constant depending $e$. In order to achieve this it must first pre-process the reference image into a search structure. This requires $O\left(n N N_{I} \log N N_{I}\right)$ time and $O\left(n N N_{I}\right)$ space.

\subsection{Relighting}

Having obtained an albedo map for a frame of the dynamic sequence a normal map is required to complete the requirements for relighting. We follow the approach of P. Csakany [15] for normal estimation. Instead of following the assumption of uniform colour regions we are able to divide out the reflectance from the original video image using our albedo map; giving a shading map $S$.

$$
S(i, j, t)=\frac{D_{l}(i, j, t)}{a_{i j t}}=\int_{\vec{\phi}} \vec{L}_{i j t}(\vec{\phi})\left(\vec{n}_{i j t} \cdot \vec{\phi}\right) d \vec{\phi}
$$

Equation 8 forms the basis of Csakany's approach [15]. It is based on the assumption that the surface has uniform Lambertian reflectance, is lit by a known point light source from the direction $\vec{L}$ and has a continuous convex form.

$$
\begin{gathered}
G(i, j, t)=\nabla S(i, j, t)-(\nabla S(i, j, t) \cdot \vec{L}) \vec{L} \\
\vec{n}(i, j, t)=\frac{S(i, j, t)}{S_{M A X}} \vec{L}+\sqrt{1-\left(\frac{S(i, j, t)}{S_{M A X}}\right)^{2}} \frac{G(i, j, t)}{\|G(i, j, t)\|}
\end{gathered}
$$

The algorithm is a 3 stage process. The first stage estimates the effective light source position by estimating the normals with the above shape from shading formula and finding the rotation between these and the normals of a prior model. Next, the normals are re-estimated using the new light position before being merged with the prior model using a weighted average.

We have briefly looked at estimating the surface normals in the same way as our albedo estimation. Initial results have been discontinuous and noisy. We believe this to be caused by a combination of lack of spatial coherence between retrieved values from $\mathbb{I}$ and the more complex relationship between surface shape and appearance not being effectively modelled by $\mathbb{I}$.

For relighting we assume point light sources and Lambertian reflectance allowing the relit image to be calculated directly from the normal map, albedo map and light position using 
Equation 9.

$$
D_{r}(i, j, t)=\sum_{m=1}^{n} A(i, j, t)\left(\vec{n}_{i j t} \cdot \overrightarrow{L_{m}}\right)+k_{a} A(i, j, t)
$$

Where $D_{r}(i, j, t)$ is the relit image, $k_{a}$ is the amount of ambient light, $L_{m}$ is the $m$ th light source direction and $n$ is the number of light sources.

\section{Results and Evaluation}

To evaluate our method we integrated our method into a capture session using the multi-view 3D capture method presented in [17]. The multi-view 3D capture made use of 8 highdefinition studio cameras and 9 standard-definition cameras running at 25fps; evenly spaced at one height around the capture volume. We set up our photo-metric stereo setup as described in Section 4.1 around one of the high-definition cameras. Several performances by an actor were captured with 2 difference costumes. We captured a reference set for the two costumes consisting of a single pose each, though the use of more poses could be expected to give improved results.

All the results presented here for our method were generated using a patch width of 11 pixels for the best match search; corresponding to a square approximately $2 \mathrm{~cm}$ across on a surface facing the camera.

Section 5.1 presents a comparative evaluation of our approach with two other methods, before which we show some sample results.

In Figure 3 we show images from a sequence relit with a point light source along with the original images from the source video. The sequence was generated using the reference set shown in Figure 2.

\subsection{Comparative Evaluation}

We evaluate our approach in comparison with segmentation and a de-lighting based approaches to reflectance estimation with the aim of comparing our approach to to those presented in $[15,10]$.

\section{De-lighting}

During the performance the studio is lit with tube lighting with tubes covering the entire ceiling, giving an approximation of ambient lighting. In these condition the main variation in illumination comes from the orientation of the surface relative to the ceiling. With surfaces facing the ceiling being lit the brightest whilst those facing progressively downwards are less brightly lit and have an increasing blue cast from he blue screening used in the studio. We model this with a shading map $s\left(n_{y}\right)$ where $n_{y}$ is the $y$ component of the surface normal. We implement this as a look up table populated with data from an image of a diffuse tube positioned horizontally under the studio lights, giving full coverage of $n_{y}$. Then for diffuse material we
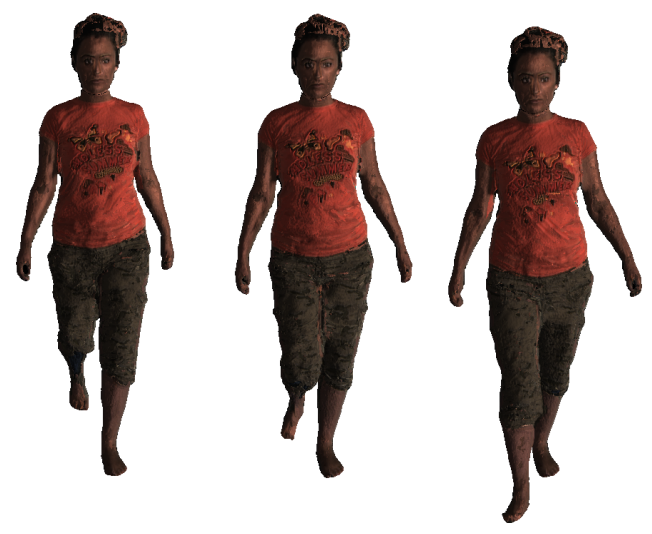

(a) Relit with point light source.
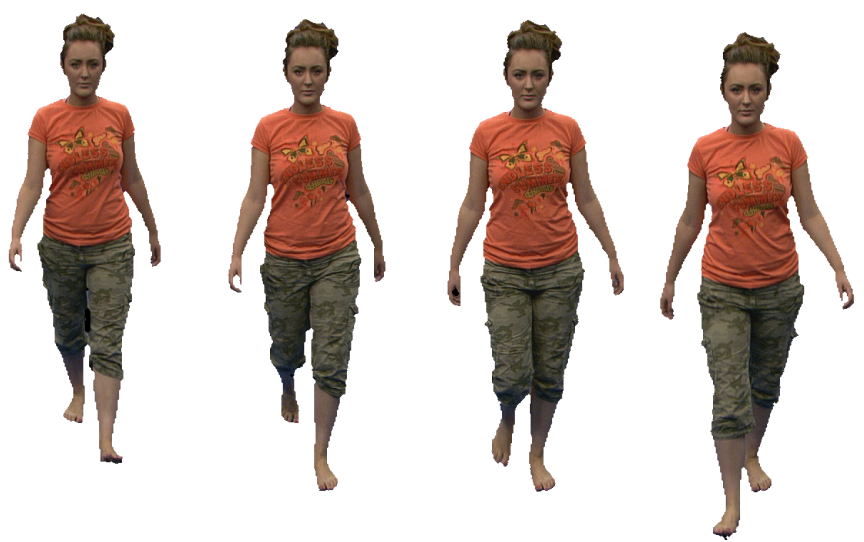

(b) Origional video.

Figure 3: Relit sequence. 
can de-light using the formula in Equation 10

$$
D_{a}(i, j, t)=\frac{D_{l}(i, j, t)}{s\left(n_{y}(i, j, t)\right)}
$$

\section{Segmentation Reflectance}

For the segmentation approach we manually segmented each colour region and assigned each region a diffuse albedo as the mean colour of the region in the video frame. The original method presented in [15] uses an automatic spatio-temporal segmentation allowing video to be coherently segmented, but for our evaluation on a small set of frames it was simpler to use a manual approach.

\section{Evaluation}

Table 1 gives a summary of the assumptions and results of the 3 methods plus texture mapping. Comparing assumptions we can see that our method is no more restricted by assumptions then the other methods, whilst being able to separate the shape detail from the reflectance.

Figure 4 gives a comparison of estimated normal maps, albedo maps and relit images. For the delit texture approach the largescale shape features captured by the multi-view reconstruction are relit but the fine-scale details such as creases remain static through out the different illumination conditions. The segmentation approach captures detailed shape detail allowing good relighting of fine creases as well as the large-scale shape. Whilst the albedo map does not capture any of the texture details leaving these to contaminate the normal map. Our approach gives the benefits of both the other two with detailed texture in the albedo map and fine-scale shape detail captured in the normal map. The creases are more subtle for our approach compared to the segmentation approach. This because it's input images to the SFS normal refinement have a lower gradient range which affects the merging between raw SFS normals and the multi-view normals. The delit texture approach looks more natural then our new approach due to artefacts in our albedo estimation. These occur mainly on the challenging reflectance of the hair and on the arms due to region edge effects and specular reflectance on the arms. The colour difference between the results for our method and the others is due to differences in illumination colour between the reference albedo set and the dynamic sequence.

In Figure 5 is an enlarged example of the albedo estimates, showing how our approach is able estimate albedo with reduced contamination from shape data without losing fine texture details.

Figure 6 shows a a relit example for a textured area. It shows how the segmentation approach fails for detailed texture whilst our method is able to handle both the fine shape and texture detail.

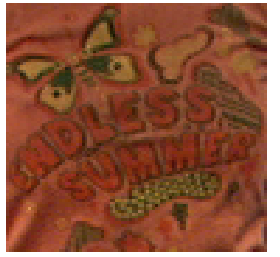

(a) Delit texture.

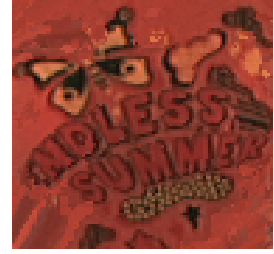

(b) Our approach.
Figure 5: Texture detail albedo comparison. (a) Albedo map contaminated with shading from creases. (b) Most creases removed.

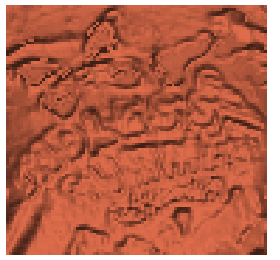

(a) Segmentation[15]

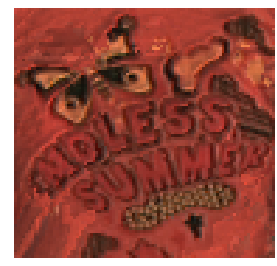

(b) Our Approach
Figure 6: Texture detail relit comparison. contaminated by texture. (b) Correctly relit.

(a) Shape detail

\section{Conclusion and Future Work}

We have presented a new approach to reflectance estimation for moving people which allows simultaneous capture of shape and reflectance detail without any special illumination conditions. We have shown that it is an improvement over previous methods in the capture of textured and patterned surfaces and in the capture of fine surface detail. However, artefacts in the reflectance estimate reduce the quality of results, suggesting further work on robustness is required.

Areas for future work to improve the robustness of our approach could be the use of temporal and spatial consistency cues or a coarse to fine approach in the best match search as well as the exploration of the use of more complex reflectance models. Other possible extensions would be to investigate applying our approach to multiple-views and the use of the reference data set to constrain the normal estimation.

\section{Acknowledgements}

We would like to thank the EPSRC and the BBC who have supported this work though a doctoral training grant and studentship.

\section{References}

[1] S. Arya, D. Mount, N. Netanyahu, R. Silverman, and A. Wu. An optimal algorithm for approximate nearest neighbor searching in fixed dimensions. ACM, (45):891923, 1998

[2] S. Barsky and M. Petrou. The 4-source photometric stereo 

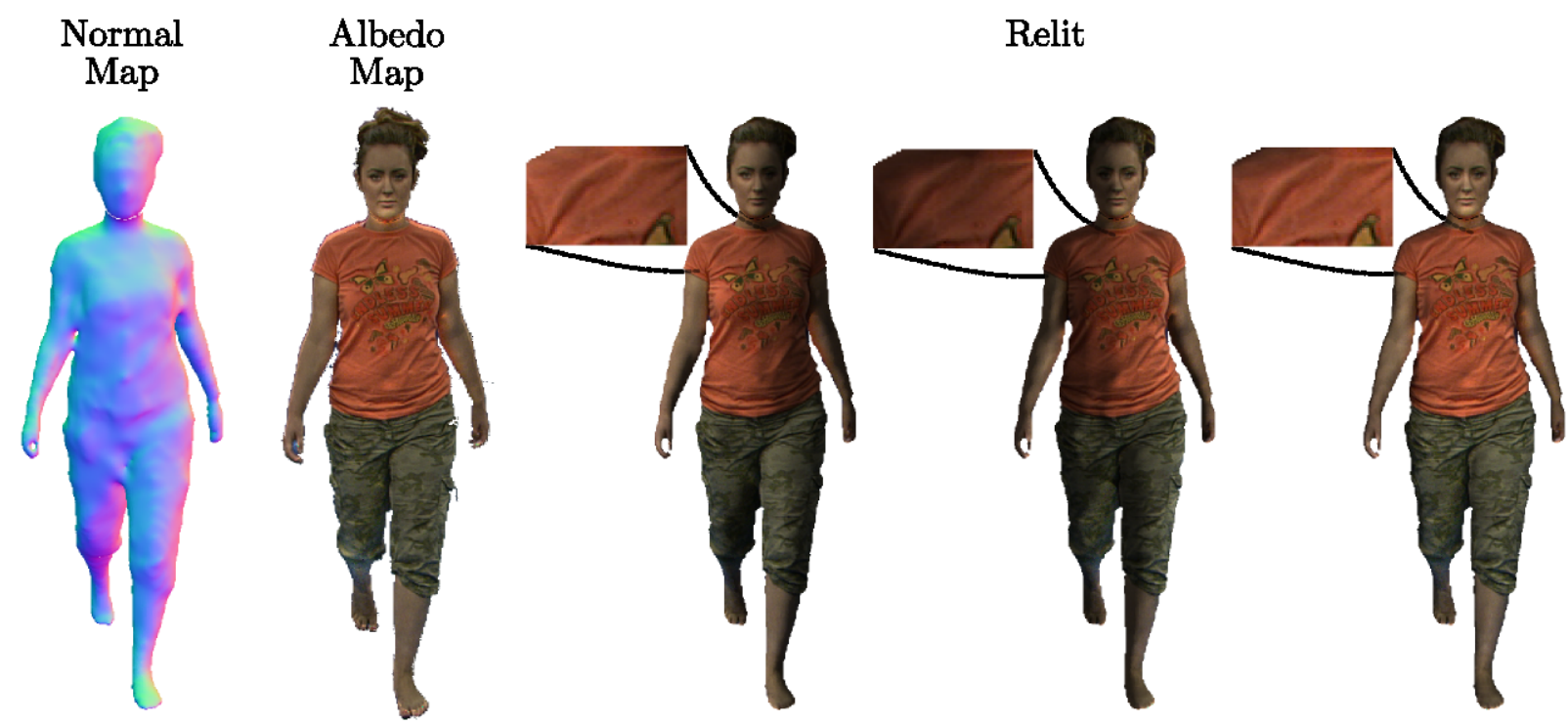

(a) Delit texture with normals from multi-view reconstruction. Similar to [10].
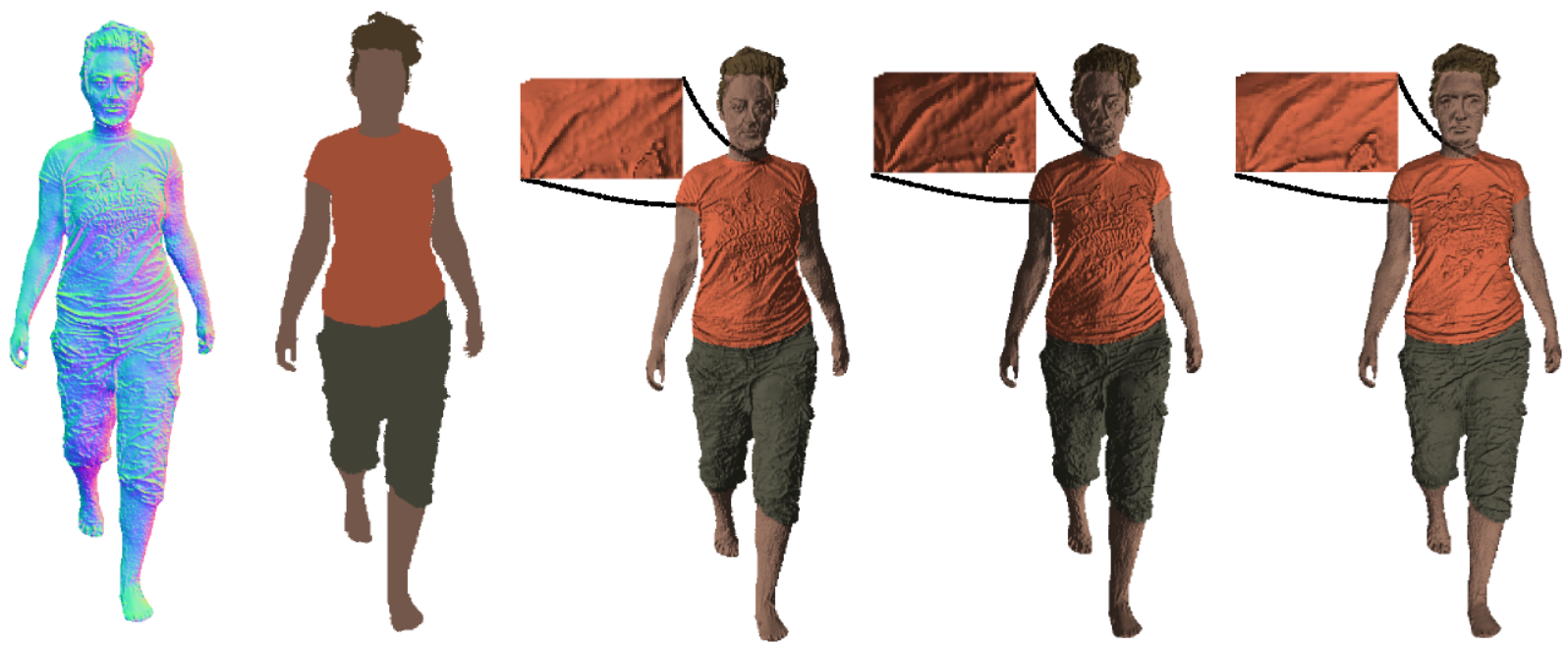

(b) Segmentation based reflectance with SFS refined normals [15].
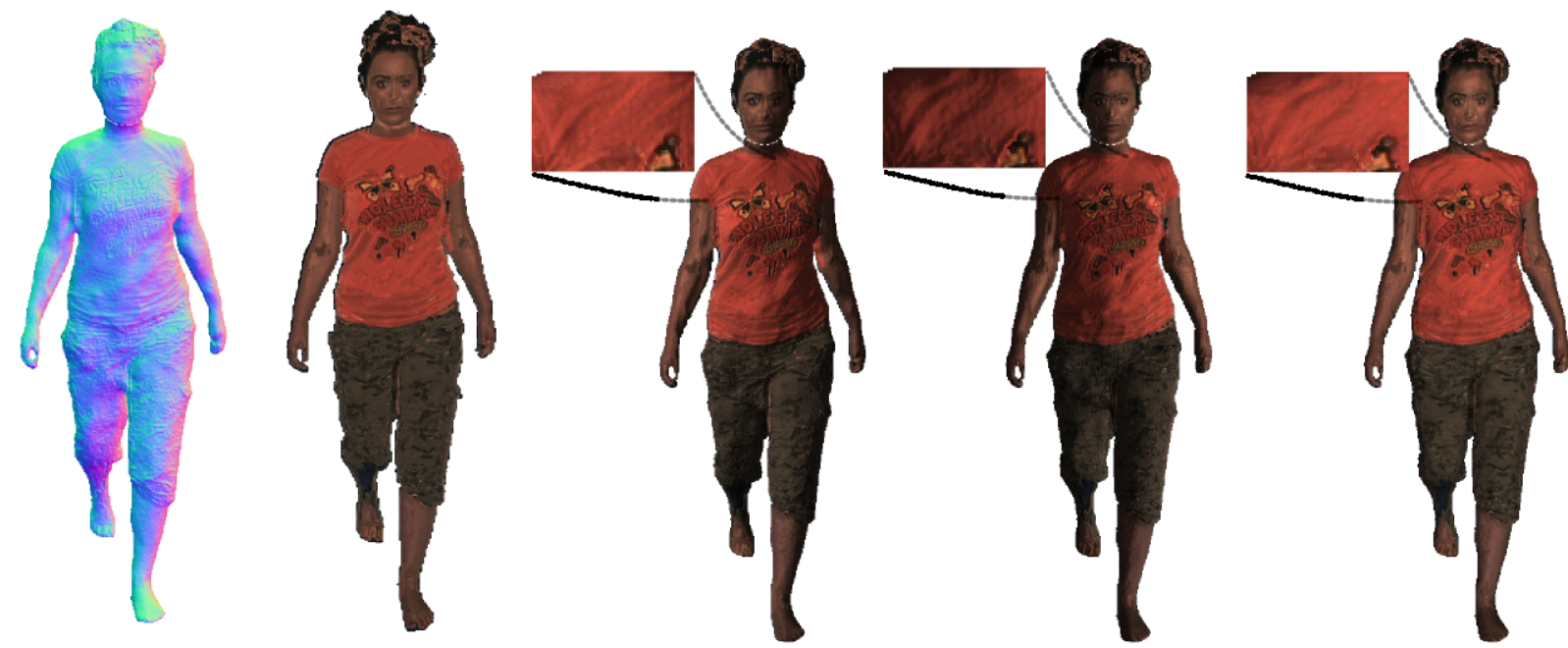

(c) Our Approach.

Figure 4: Comparison of reflectance estimation methods. The first column shows the estimated normal maps, the second shows the acquired albedo maps and the remaining columns show relit results under different illumination conditions. (a) Creases do not respond to changing light position. (b) Dynamic lighting of creases but texture contaminates shape data instead of being captured in reflectance map. (c) Dynamic lighting of creases with texture detail separated from shape detail. 


\begin{tabular}{|l|c|c|c|c|}
\hline Method & Texture [17] & Delit Texture [10] & Segmentaion [15] & Ours \\
\hline \hline Assumptions & & & & \\
\hline \hline Known Illumination & $\times$ & $\sqrt{ }$ & $\times$ & $\times$ \\
\hline Uniform Albedo Regions & $\times$ & $\times$ & $\sqrt{ }$ & $\times$ \\
\hline Lambertian Reflectance & $\times$ & $\sqrt{ }$ & $\sqrt{ }$ \\
\hline \hline Results & & & & \\
\hline \hline Separation of Reflectance from Shape & $\times$ & $\times$ & $\times$ & $\sqrt{ }$ \\
\hline Large-scale Relighting & $\times$ & $\sqrt{ }$ & $\sqrt{ }$ \\
\hline Fine-scale Relighting & $\times$ & $\times$ & $\sqrt{ }$ \\
\hline
\end{tabular}

Table 1: Comparison of dynamic relighting approaches.

technique for three-dimensional surfaces in the presence of highlights and shadows. Pattern Analysis and Machine Intelligence, IEEE Transactions on, 25, Issue 10:12391252, Oct. 2003.

[3] P. Csakany and A. Hilton. Relighting of facial images. In Automatic Face and Gesture Recognition, 2006. FGR 2006. 7th International Conference on, pages $55-60$, April 2006.

[4] A. Efros and T. Leung. Texture synthesis by nonparametric sampling. In IEEE International Conference on Computer Vision, September 1999.

[5] Alexei A. Efros and William T. Freeman. Image quilting for texture synthesis and transfer. In Eugene Fiume, editor, SIGGRAPH 2001, Computer Graphics Proceedings, pages 341-346. ACM Press / ACM SIGGRAPH, 2001.

[6] P. Einarsson, C. Chabert, A. Jones, W. Ma, B. Lamond, T. Hawkins, M. Bolas, S. Sylwan, and P. Debevec. Relighting human locomotion with flowed reflectance fields. In Rendering Techniques 2006: 17th Eurographics Workshop on Rendering, pages 183-194, June 2006.

[7] H. Fang and J. C. Hart. Textureshop: texture synthesis as a photograph editing tool. ACM Transactions on Graphics, 23(3):354-359, aug 2004.

[8] H. Fang and J.C. Hart. Rototexture: Automated tools for texturing raw video. Visualization and Computer Graphics, IEEE Transactions on, 12(6):1580 - 1589, Nov.-Dec. 2006.

[9] A.W. Fitzgibbon, Y. Wexler, and A. Zisserman. Imagebased rendering using image-based priors. In ICCV, 2003.

[10] O. Grau. Multi-camera radiometric surface modelling for image-based re-lighting. In DAGM-Symposium, pages 667-676, 2006.

[11] F. Han and S. Zhu. A two-level generative model for cloth representation and shape from shading. Pattern
Analysis and Machine Intelligence, IEEE Transactions on, 29:1230 - 1243, July 2007.

[12] C. Hernández, G. Vogiatzis, G.J. Brostow, B. Stenger, and R. Cipolla. Non-rigid photometric stereo with colored lights. In Computer Vision, 2007. ICCV 2007. IEEE 11th International Conference on, pages 1 - 8, Oct. 2007.

[13] A Hertzmann, C. Jacobs, N. Oliver, B. Curless, and D. Salesin. Image analogies. In SIGGRAPH 2001 Conference Proceedings.

[14] E. A. Khan, E. Reinhard, R. W. Fleming, and H. H. Bulthoff. Image-based material editing. ACM Transactions on Graphics, 25(3):654-663, jul 2006.

[15] F. Vajda P. Csakany and A. Hilton. Recovering refined surface normals for relighting clothing in dynamic scenes. In CVMP 2007, 2007.

[16] Y. Sato, M. D. Wheeler, and K. Ikeuchi. Object shape and reflectance modeling from observation. Computer Graphics, 31(Annual Conference Series):379-388 (or 379-387??), 1997.

[17] J. Starck and A. Hilton. Surface capture for performance based animation. IEEE Computer Graphics and Applications, 27(3):21-31, 2007.

[18] C. Theobalt, N. Ahmed, H. Lensch, M. Magnor, and H. Seidel. Seeing people in different light-joint shape, motion, and reflectance capture. Transactions on Visualization and Computer Graphics, 13, Issue 4:663 674, July-Aug. 2007.

[19] O. Woodford and A. Fitzgibbon. Fast image-based rendering using hierarchical image-based priors. In 16th BMVC Proceedings. BMVA, September 2005. 